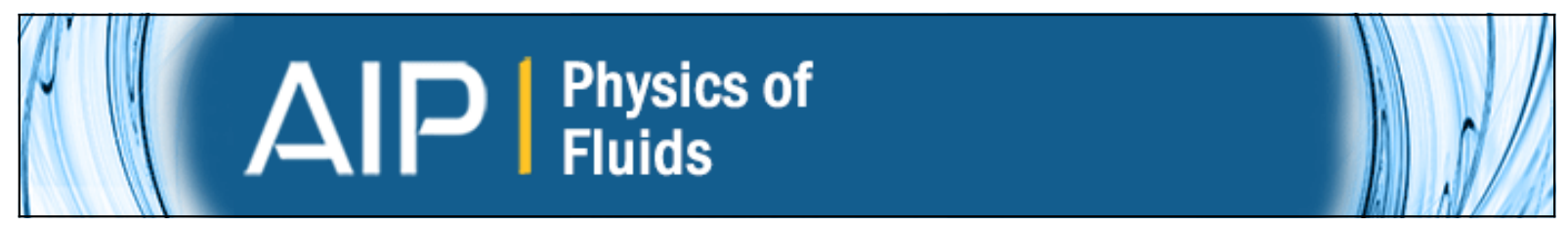

\title{
Unsteady asymmetric engulfment regime in a T-mixer
}

Andrea Fani, Simone Camarri, and Maria Vittoria Salvetti

Citation: Physics of Fluids (1994-present) 26, 074101 (2014); doi: 10.1063/1.4885451

View online: http://dx.doi.org/10.1063/1.4885451

View Table of Contents: http://scitation.aip.org/content/aip/journal/pof2/26/7?ver=pdfcov

Published by the AIP Publishing

Articles you may be interested in

Direct numerical simulation of electrokinetic instability and transition to chaotic motion

Phys. Fluids 25, 122001 (2013); 10.1063/1.4843095

A lift formula applied to low-Reynolds-number unsteady flows

Phys. Fluids 25, 093605 (2013); 10.1063/1.4821520

Low-frequency unsteadiness in the vortex formation region of a circular cylinder

Phys. Fluids 25, 085109 (2013); 10.1063/1.4818641

Investigation of the steady engulfment regime in a three-dimensional T-mixer

Phys. Fluids 25, 064102 (2013); 10.1063/1.4809591

Nonlinear regime of a multimode Richtmyer-Meshkov instability: A simplified perturbation theory

Phys. Fluids 14, 1111 (2002); 10.1063/1.1447914

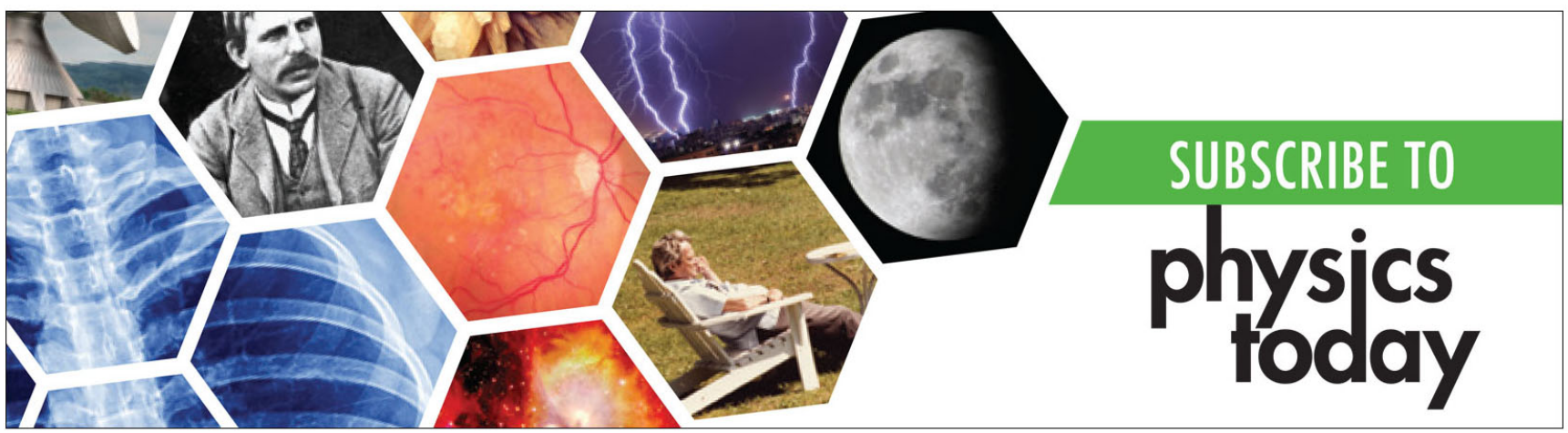




\title{
Unsteady asymmetric engulfment regime in a T-mixer
}

\author{
Andrea Fani, ${ }^{1}$ Simone Camarri, ${ }^{2, a)}$ and Maria Vittoria Salvetti ${ }^{2}$ \\ ${ }^{1}$ Laboratory of Fluid Mechanics and Instabilities, École Polytechnique Fédérale de \\ Lausanne, Lausanne CH-1015, Switzerland \\ ${ }^{2}$ Dipartimento di Ingegneria Civile ed Industriale, Università di Pisa, via G. Caruso 8, 56122 \\ Pisa, Italy
}

(Received 22 March 2014; accepted 14 June 2014; published online 2 July 2014)

\begin{abstract}
Unsteady flow regimes in a micro T-mixer are investigated. Direct numerical simulations (DNS) show that, in agreement with experimental and numerical results in the literature, when the Reynolds number is increased above a critical value larger than those typical of the steady engulfment regime, the flow remains asymmetric in the mean but becomes periodic in time. The dynamics of this regime is characterized by analyzing the evolution of the three-dimensional vortical structures forming at the confluence of the two inlet flow streams. As the Reynolds number is further increased, the flow remains time-periodic but it continuously switches between a symmetric configuration, similar to that of the vortical regime, and an asymmetric one, close to the engulfment configuration. Three-dimensional linear stability analysis is successively used to characterize the instability leading to the unsteady asymmetric regime, which is also interesting for applications due to its high mixing efficiency. The critical Reynolds number and the instability frequency are in very good agreement with those found in DNS. The sensitivity of this instability to a generic perturbation of the base flow is also investigated. It is shown that the largest sensitivity is to base-flow modifications introduced close to the three-dimensional vortical structures forming at the confluence between the inlet channels. Finally, the sensitivity analysis is specialized to investigate the effect of a generic perturbation of the inlet velocity profile. (C) 2014 AIP Publishing LLC. [http://dx.doi.org/10.1063/1.4885451]
\end{abstract}

\section{INTRODUCTION}

T-shaped micromixers are commonly used in microfluidics to promote mixing between two fluid streams; in this type of applications, two streams enter from the two opposite channels (inlet channels) and the mixture exit from the third channel (outflow channel). Such configurations are also often found as junction elements in complex micro-systems.

The flow in micro T-mixers is characterized by different regimes, whose onset depends on different parameters, such as the Reynolds number, the mixer geometry, and the inflow conditions.

Most of the studies available in literature focused their attention on the engulfment, which is a steady regime occurring when the Reynolds number exceeds a given critical threshold. This regime is characterized by a loss of the flow symmetries in the outflowing channel and by a convectively driven mixing between the two flows. This leads to a considerable mixing efficiency, which motivates the interest in the literature for the engulfment regime (ER) (see, e.g., Refs. 1-9). Geometrical parameters such as the width ratio between the outflowing and inlet channels $W_{o} / W$ and the width to height ratio $W_{o} / H$ have been found to have a significant impact on the value of the critical Reynolds number for engulfment regime (see, e.g., the review by Santos and Sultan ${ }^{10}$ and the references therein).

More recently, an unsteady periodic regime was identified in numerical simulations and experiments for Reynolds numbers moderately larger than the engulfment critical one. Dreher et al. ${ }^{11}$

\footnotetext{
a)s.camarri@ing.unipi.it
} 
simulated the flow in a T- shaped micro-mixer with square inlet channels and an outflow channel having a width to height ratio $W_{o} / H=2$. They found for $R e>240$ the onset of an unsteady regime, where $R e$ is the Reynolds number based on the outflow hydraulic diameter and on the bulk inlet velocity. They identified a periodic pulsating flow for $240<R e<400$ characterized by a Strouhal number (based, as $R e$, on the outflow hydraulic diameter and on the bulk inlet velocity) $0.15<S t<0.29$. Such periodic pulsations were found to disappear for larger Re, i.e., when $\operatorname{Re}>500$, where a chaotic motion was observed. Their numerical results were corroborated by some experimental flow visualizations and by the results of more recent direct numerical simulations ${ }^{12}$ (DNS) of the same configuration, in which an unsteady periodic flow regime was also detected for a Reynolds number range in full agreement with that indicated by Dreher et al. ${ }^{11}$ Galletti et al. ${ }^{6}$ numerically investigated the flow behavior in a T-mixer with a rectangular inlet channel $(W / H=0.75$, where $W$ and $H$ are the inlet channel width and height, respectively) up to $R e=480$. For fully developed inlet velocity conditions, they found an unsteady pulsating regime at $288 \leq R e \leq 393$. The degree of mixing in such conditions was found to be larger than that observed for the steady flow at lower Reynolds numbers. For a non-fully developed inlet velocity profile, the unsteady regime was not detected at any of the investigated Reynolds numbers (up to $R e=527$ ).

Unsteady periodic regimes were also detected experimentally and analyzed by Thomas et al. ${ }^{13,14}$ for the same T-mixer geometry as in Dreher et al. ${ }^{11}$ Thomas et al. ${ }^{13,14}$ distinguished between two different unsteady flow regimes. First, an unsteady periodic asymmetric flow was found for $260 \leq$ $R e<460$. In this regime, Thomas et al. ${ }^{13,14}$ observed two or four vortical structures periodically forming at the T-channel junction, which they attributed to the roll-up of the shear layers present in this zone, and the generation of periodic coherent waveforms in the outlet channel. By further increasing the Reynolds number $(R e \geq 467)$, the flow was found to remain unsteady, but characterized by a symmetric four-vortex topology, in which the stagnation point oscillates. They found this latter regime detrimental to mixing, although bursts of improved mixing may occur as a consequence of losses of symmetry intermittently occurring in the flow.

In the present work, we focus on the unsteady asymmetric flow regime, which is particularly interesting from the viewpoint of mixing efficiency. DNS and global stability and sensitivity analyses are used together to bring further insight on the onset, on the dynamics, and on the features of the unsteady asymmetric flow regime. To this aim, the same configuration as in Galletti et al. ${ }^{6}$ and Fani et $a l .{ }^{8}$ is considered and the same methodology as in Fani et al. ${ }^{8}$ is used. This latter work was focused on the investigation of the onset and of the characteristics of the steady engulfment regime. The dynamics of the three-dimensional (3D) vortical structures in the different regimes is characterized herein by means of DNS, especially highlighting their modifications when passing from a regime to another, viz., from steady engulfment to unsteady asymmetric engulfment and eventually to the unsteady symmetric (USR)/asymmetric regime (UAR). This investigation is complemented by linear global stability and sensitivity analyses, focused on the onset of the unsteady asymmetric regime. Global stability analysis provides an accurate estimation of the critical Reynolds numbers for the onset of this flow regime, together with the identification of the spatial distribution and frequency of the associated unstable mode. Furthermore, the instability core, i.e., the region where instability originates, can be detected, as well as the nature of the instability can be highlighted. Finally, the sensitivity of the detected instability to a localized flow perturbation is investigated based on adjoint methods. ${ }^{8,15-17}$ This gives quantitative information which can be used in possible control strategies aimed at promoting or delaying the onset of unsteady asymmetric regime. As an example, we provide herein a systematic analysis of the sensitivity to a perturbation of the inlet velocity conditions. This is of interest for applications because, for practical size limitations, the velocity profiles in the inlet channels may significantly differ from fully developed conditions. A similar analysis, carried out in Fani et al., ${ }^{8}$ showed that inlet velocity conditions have a remarkable influence on the onset of the instability leading to steady engulfment, while in the DNS in Galletti et al. ${ }^{6}$ a significant influence was also observed on the onset of the unsteady regime. Note that the sensitivity maps shown in the present paper can also be used to devise different control strategies. As a final remark, to the authors' knowledge, the present study is the first example of the application of fully 3D global stability and sensitivity analyses to the onset of the instability leading to the unsteady regime in a T-mixer, as well as the work of 


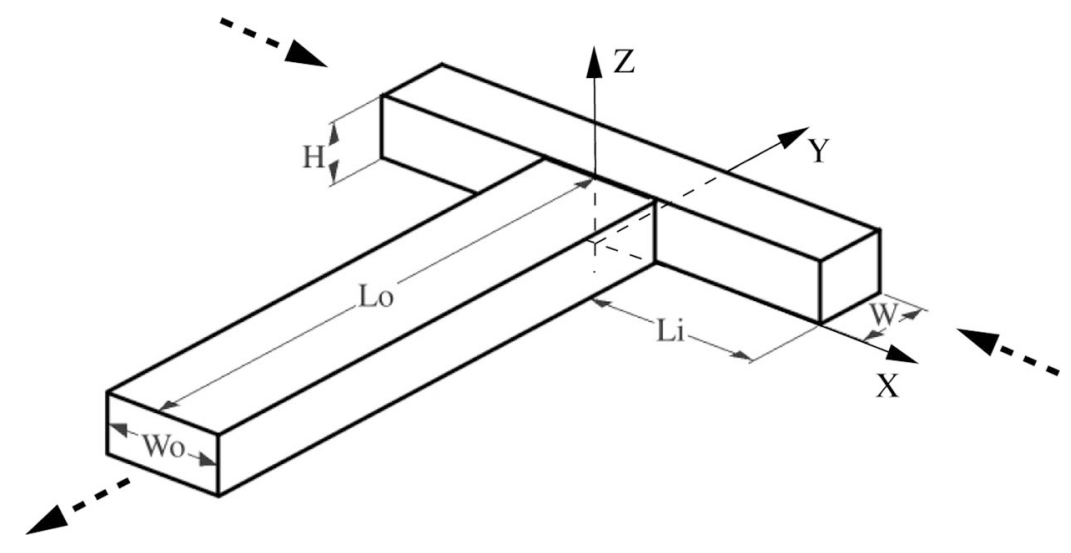

FIG. 1. Flow configuration and frame of reference; dashed lines indicate the flow direction.

Fani et $a l .{ }^{8}$ provided the first example of application of the same techniques to steady engulfment regime.

\section{PROBLEM DESCRIPTION AND METHODOLOGY}

The considered T-mixer geometry is made by two inflow and one outflow pipes, all with rectangular cross sections. The flow is incompressible and the working fluid is Newtonian. All quantities are normalized using the hydraulic diameter $D_{h}$ of the outflowing pipe and the bulk velocity of the inlet flow $U_{m}$. Therefore, the Reynolds number $R e$ is defined as $R e=U_{m} D_{h} / \nu$. The T-mixer geometry and the frame of reference are sketched in Fig. 1 (not in scale). The height of the mixer is $H \simeq 0.83$, the width of the incoming pipes is $W=0.625$, and the width of the outcoming pipe is $W_{o}=2 W=1.25$, the aspect ratio of the incoming and outcoming cross sections being equal to $W / H=0.75$ and $W_{o} / H=1.50$, respectively. The lengths of the inflow and outflow pipes are $L_{i}$ $=6.875$ and $L_{o}=25$. The flow configuration is the same investigated in Galletti et al..$^{6}$ and Fani et al. ${ }^{8}$ except for the length of the outflow pipe $L_{o}$, which here is the double of that used in the previous works. Indeed, the reduced computational domain $\left(L_{o}=12.5\right)$ was found inadequate to simulate correctly the flow dynamics in the considered range of Reynolds numbers, in particular for the largest ones, where the complex vortical structures extend further in the mixing channel.

The fluid motion is described by the three-dimensional unsteady incompressible Navier-Stokes equations

$$
\begin{aligned}
& \frac{\partial \mathbf{U}}{\partial t}+\mathbf{U} \cdot \nabla \mathbf{U}+\nabla P-\frac{1}{R e} \nabla^{2} \mathbf{U}=\mathbf{0}, \\
& \nabla \cdot \mathbf{U}=0,
\end{aligned}
$$

where $\mathbf{U}=(U, V, W)$ is the velocity vector and $P$ is the reduced pressure. No-slip boundary conditions are imposed at the mixer walls, while at the inlet a unidirectional fully developed velocity profile is assumed. ${ }^{1}$ Finally, free outflow conditions of the following type are used: $\partial_{y} U=0, P-R e^{-1} \partial_{y} V=0, \partial_{y} W=0$.

For linear stability analysis, the flow variables are decomposed in the base flow $\left(\mathbf{U}_{b}, P_{b}\right)$, steady solution of Eqs. (1), and in an unsteady perturbation $(\mathbf{u}, p)$. The perturbation is searched in the form of normal modes $\mathbf{q}(x, y, z, t)=(\mathbf{u}, p)(x, y, z, t)=(\hat{\mathbf{u}}, \hat{p})(x, y, z) \exp (\sigma t)$, where $\sigma$ is a generally complex eigenvalue associated with the mode $(\hat{\mathbf{u}}, \hat{p})$. Introducing the flow decomposition and the normal-mode form into Eqs. (1) and linearizing in the disturbance, we obtain the following equations governing the dynamics of the perturbation:

$$
\begin{aligned}
& \sigma \hat{\mathbf{u}}+\hat{\mathbf{u}} \cdot \nabla \mathbf{U}_{b}+\mathbf{U}_{\mathbf{b}} \cdot \nabla \hat{\mathbf{u}}+\nabla \hat{p}-\frac{1}{R e} \nabla^{2} \hat{\mathbf{u}}=\mathbf{0}, \\
& \nabla \cdot \hat{\mathbf{u}}=0 .
\end{aligned}
$$


The boundary conditions associated with Eqs. (2b) are: $\hat{\mathbf{u}}=0$ at the inlet surfaces and on the walls, and free outflow conditions at the outlet. Equations (2b) along with the boundary conditions are an eigenvalue problem. The flow is linearly stable when all the eigenvalues, $\sigma=\lambda+1 \omega$, are characterized by $\lambda<0$.

The main steps needed to compute the sensitivity of the considered instability to a modification of the boundary conditions, acting at base-flow level, are the following. First, we consider a generic base-flow modification $\delta \mathbf{U}_{b}$. The modification $\delta \sigma$ of the eigenvalue caused by this arbitrary variation of the base flow can be written as follows: $:^{15,17}$

$$
\delta \sigma=\frac{\left\langle M^{+}, \delta \mathbf{U}_{b}\right\rangle}{\left\langle\hat{\mathbf{u}}^{+}, \hat{\mathbf{u}}\right\rangle},
$$

where $\langle a, b\rangle=\int_{\Omega}\left(a^{*} \cdot b\right) d \Omega$ is the scalar product between the complex vectors $a$ and $b$ on the flow domain $\Omega$ and the asterisk $*$ denotes the conjugate of a complex quantity. $M^{+}$is the sensitivity vector field, which has the following expression:

$$
M^{+}=\hat{\mathbf{u}}^{*} \cdot \nabla \mathbf{u}^{+}-\nabla \hat{\mathbf{u}}^{*} \cdot \mathbf{u}^{+},
$$

where $\mathbf{u}^{+}$is the velocity part of the mode adjoint to $(\hat{\mathbf{u}}, \hat{p}, \sigma)$, solution of the following eigenvalue problem:

$$
\begin{aligned}
& \sigma^{*} \mathbf{u}^{+}+\nabla \mathbf{U}_{b} \cdot \mathbf{u}^{+}-\mathbf{U}_{\mathbf{b}} \cdot \nabla \mathbf{u}^{+}+\nabla p^{+}-\frac{1}{R e} \nabla^{2} \mathbf{u}^{+}=\mathbf{0}, \\
& \nabla \cdot \mathbf{u}^{+}=0 .
\end{aligned}
$$

The boundary conditions associated with problem $(5 b)$ are: $\mathbf{u}^{+}=0$ at the inlet surfaces and on the lateral walls, and $p^{+} \mathbf{n}-R e^{-1}\left(\mathbf{n} \cdot \nabla \mathbf{u}^{+}\right)=\left(\mathbf{U}_{\mathbf{b}} \cdot \mathbf{n}\right) \mathbf{u}^{+}$at the outlet. ${ }^{17}$ Finally, the following condition is used to normalize the adjoint velocity field:

$$
\left\langle\hat{\mathbf{u}}^{+}, \hat{\mathbf{u}}\right\rangle=1 .
$$

A perturbation of the velocity distribution $\left(\delta \mathbf{U}_{\mathbf{i}}\right)$ at the inlet surfaces, which is a boundary condition for the base-flow problem, induces a base flow modification $\delta \mathbf{U}_{b}$ and, thus it is possible to quantify the perturbation effect on the instability using Eq. (3). Nonetheless, this implies the computation of specific base-flow modifications for each considered inlet perturbation. Following the approach of Marquet et al., ${ }^{17}$ the eigenvalue drift caused by a generic perturbation $\delta \mathbf{U}_{\mathbf{i}}$ of the inlet boundary conditions can be computed as follows:

$$
\delta \sigma=\frac{\left\langle P_{b}^{+} \mathbf{n}+R e^{-1} \mathbf{n}^{T} \cdot \nabla \mathbf{U}_{\mathbf{b}}^{+}, \delta \mathbf{U}_{\mathbf{i}}\right\rangle_{i}}{\left\langle\mathbf{u}^{+}, \hat{\mathbf{u}}\right\rangle},
$$

where the subscript $i$ indicates a scalar product calculated on the inlet boundary of the domain, i.e., $\langle a, b\rangle_{i}=\int_{\Gamma_{i}}\left(a^{*} \cdot b\right) d l$.

In Eq. (7), $\mathbf{U}_{\mathbf{b}}{ }^{+}$and $P_{b}^{+}$are, respectively, the adjoint base flow velocity and the adjoint pressure, solution of the following problem:

$$
\begin{aligned}
& \nabla \mathbf{U}_{\mathbf{b}} \cdot \mathbf{U}_{b}^{+}-\mathbf{U}_{\mathbf{b}} \cdot \nabla \mathbf{U}_{\mathbf{b}}^{+}+\nabla P_{b}^{+}-\frac{1}{R e} \nabla^{2} \mathbf{U}_{\mathbf{b}}^{+}=M^{+}, \\
& \nabla \cdot \mathbf{U}_{\mathbf{b}}^{+}=0,
\end{aligned}
$$

along with homogeneous boundary conditions at the inlet surfaces and on the solid walls and with the following condition on the outflow boundary: $P^{+} \mathbf{n}-R e^{-1}\left(\mathbf{n} \cdot \nabla \hat{\mathbf{U}}_{\mathbf{b}}^{+}\right)=-\left(\mathbf{U}_{\mathbf{b}} \cdot \mathbf{n}\right) \mathbf{U}_{\mathbf{b}}^{+}+\left(\hat{\mathbf{u}}^{*} \cdot \mathbf{n}\right) \hat{\mathbf{u}}^{+}$. 
The open-source massively parallel spectral element code NEK5000 ${ }^{18}$ has been used for the numerical solution of the equations concisely recalled above. The velocity space is spanned by Nthorder Lagrange polynomial interpolants, based on tensor-product arrays of Gauss-Lobatto-Legendre (GLL) quadrature points in each hexahedral element, while the polynomial order for pressure is $N-2$. Time discretization uses explicit backward-differentiation for convective terms, and an implicit scheme for viscous terms. A third order scheme (BDF3) is used for the nonlinear NavierStokes equations. The eigenvalue problem (2) and (5) have been solved by an Arnoldi iteration method ad hoc implemented by the authors in NEK5000. The linearized/adjoint version of the code has been used as a time-stepper, using a second order scheme (BDF2) for the time integration. The base flow is the asymptotic steady solution of the nonlinear simulations when the flow is stable, whereas, for Reynolds number larger than the critical one, it is obtained by using the selective frequency damping method proposed by Akervik et al. ${ }^{19} \mathrm{~A}$ validation of the numerical tools built in the framework of NEK5000 for stability and sensitivity analysis can be found in Fani et al. ${ }^{8}$

The results shown here have been obtained using a structured multi-block grid. Along the width of all the channels, a uniform element size of 0.07 is chosen, while along their axis the size varies between 0.08 and 0.5 . In the $z$ direction, the element size is uniform equal to 0.083 . The polynomial order for the velocity is equal to 7. Globally, the used grid has 15680 elements, associated to $5.2 \times 10^{6}$ degrees of freedom for the velocity. As concerns the time discretization, a constant time step equal to $7 \times 10^{-4}$ is adopted for both the nonlinear simulations and the linearized equations used in the time-stepper approach, leading to a CFL number equal to 0.3.

\section{DNS INVESTIGATION}

The simulations carried out in the present work show that the flow becomes unsteady and perfectly periodic at a critical Reynolds number in the range $220<R e_{c r 1}<230$.

In order to quantify the period of the unsteady flow, the velocity time history has been recorded at different positions along the $y$ axis, namely, at $y=0.5,-2,-5,-9,-20$. At each $R e$, the characteristic Strouhal number has been computed as the peak frequency of the time velocity spectra, normalized by the inlet bulk velocity and the outlet hydraulic diameter, $S t=\frac{f D_{h}}{U_{m}}$. This Strouhal number is shown in Fig. 2 as a function of $R e$. At the onset of the instability $S t \simeq 0.16$, and $S t$ increases together with $R e$ up to $S t \simeq 0.3$ for $R e=400$. In the range $230 \leq R e \leq 320$, the flow is perfectly periodic everywhere in the computational domain, as also confirmed by the velocity signals in all the previously mentioned locations. As $R e$ is further increased in the range $360 \leq R e<400$ the flow at the intersection of the three pipes remains periodic but, progressing towards the outflow pipe, the velocity signals show irregular fluctuations superposed to a periodic behaviour. For this reason, the values of $S t$ reported in Fig. 2 have been estimated from the velocity signals measured at $y=0.5$. Moreover, as it will be shown in Sec. III C, this location is inside the core of the global instability leading to the unsteady regime, characterized by a self-sustained periodic process, which

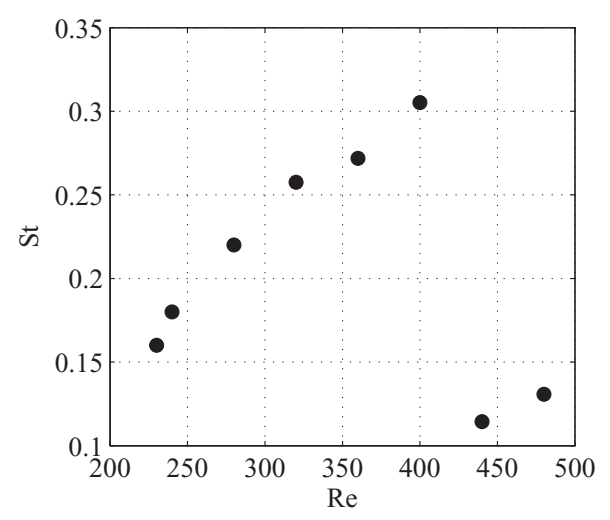

FIG. 2. DNS simulations: Strouhal number of the periodic flow regime as a function of the flow Reynolds number. 


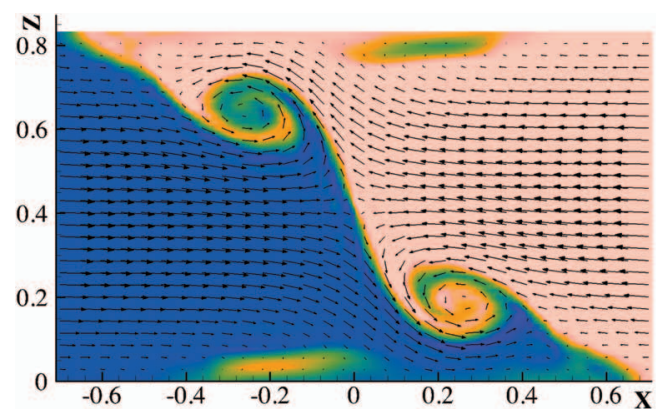

(a)

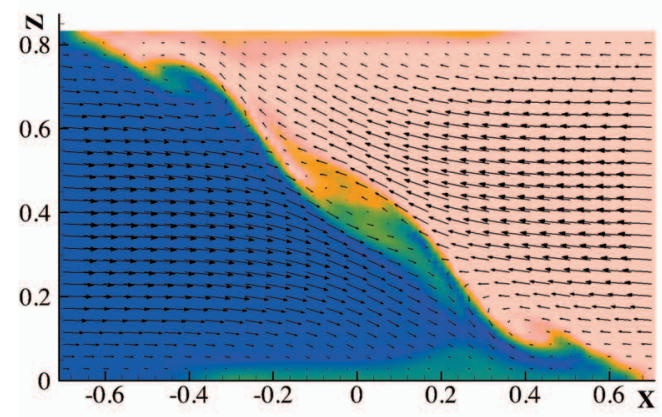

(c)

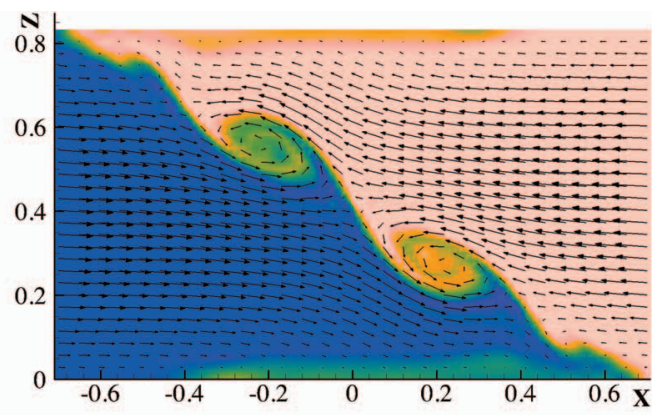

(b)

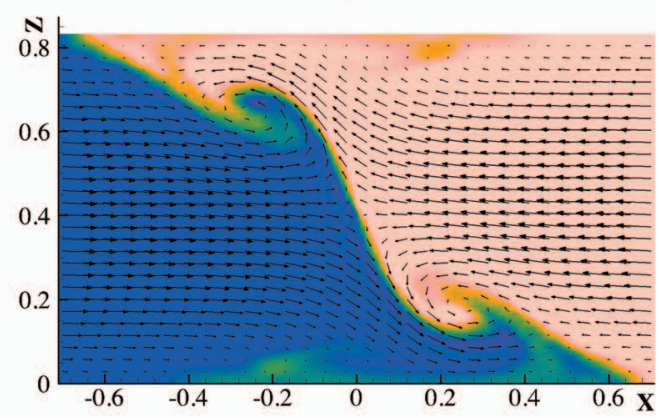

(d)

FIG. 3. Section at $y=0.31$ at $R e=240$ : in-plane velocity vectors and distribution of a passive scalar (light color), entering from one channel of the mixer, at four uniformly distributed phases in one instability cycle (from (a) to (d): $t / T=0,0.25$, $0.5,0.75, T$ being the flow period) (Multimedia view) [URL: http://dx.doi.org/10.1063/1.4885451.1]

will be described in the following. The irregular fluctuations present in the mixing channel are probably due to convective instabilities of the flow, which behaves as a noise amplifier in this region.

In summary, in the range $230 \leq R e<400$, the flow regime observed in our DNS is periodic with a characteristic $S t$ monotonically increasing with $R e$. In a similar range of Reynolds numbers, unsteady periodic flows have also been found in previous works in the literature. ${ }^{6,11,13}$ In particular, laser-induced fluorescence (LIF) visualizations of a passive scalar in the junction centerplane of a square-section T-mixer are reported in Thomas et al. ${ }^{13}$ (see Fig. 8 of the referenced paper). They show that, when the two flows entering the mixer meet, they seem to slide one over the other on an inclined ideal separating surface. Along the sliding surface vortices resembling a Kelvin-Helmholtz instability form, approach and disappear, this process being repeated periodically in time. A similar visualization has been reproduced in our DNS by simulating the dynamics of a passive scalar entering the flow from one inlet channel, in order to provide an easy visual comparison with Thomas et al. ${ }^{13}$ In the advection-diffusion equation governing the dynamics of the passive scalar, the diffusivity $k$ has been kept very low $\left(k=10^{-4}\right)$, so as to keep the boundary of the region occupied by the scalar sharp and to highlight the role of convection in the mixing process. Visualizations of the passive scalar on the symmetry plane of the inlet pipes $(y \simeq 0.31$ ) are reported in Fig. 3 (Multimedia view), where 4 frames distributed in a period are reported for $R e=240$. Although a different aspect ratio of the inlet channels than in Thomas et al. ${ }^{13}$ is considered here, the similarity with the experimental visualizations reported in the referenced paper is striking, leaving no doubts on the fact that the simulated regime is the one identified as the UAR. The dynamics of the UAR will be analyzed more in detail in Sec. III A.

As the Reynolds number is increased, Fig. 2 shows a sudden jump in the flow dominant frequency at $400<R e_{c r 2}<440$. Moreover, for Reynolds number in the range $440 \leq R e \leq 480$ the velocity signals measured everywhere in the outflow channel are again perfectly periodic. The jump in the $S t-R e$ curve is associated to another bifurcation leading to a change in the flow regime from the UAR to the unsteady symmetric flow regime. The USR has been identified by visualizations of 


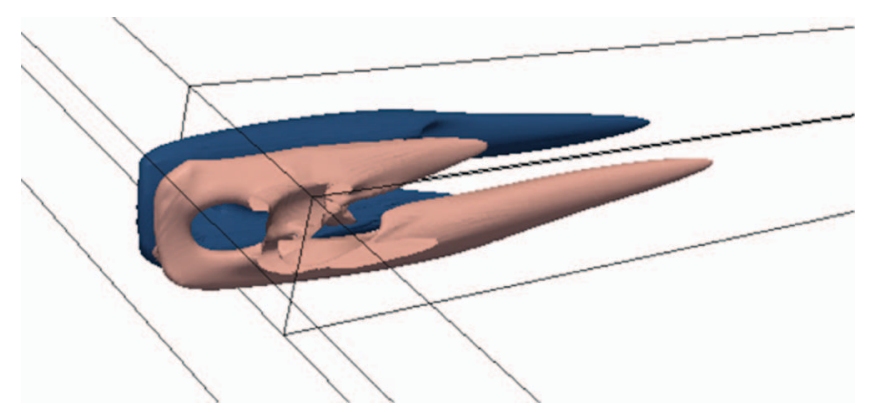

FIG. 4. Engulfment regime $(R e=160)$ : vortices identified by the $\lambda_{2}$ criterion; the color indicates the sign of the axial vorticity of each vortex.

the passive scalar in Thomas et al. ${ }^{13}$ The flow features in this regime will be briefly described in Sec. III B.

\section{A. Unsteady asymmetric regime}

As highlighted in the previous discussion and shown in Fig. 3, co-rotating vortices which resemble a Kelvin-Helmholtz instability are observable at the interface between the two flows entering the mixer. We now show that this similarity is only apparent, and that the vortices are instead strongly related to $3 \mathrm{D}$ vortical structures similar to those characteristic of the steady ER. For a detailed description of the ER, we refer to Fani et al. ${ }^{8}$ In the following, the vortical structures are identified though the $\lambda_{2}$ criterion proposed by Jeong and Hussain, ${ }^{20} \lambda_{2}$ is the second largest eigenvalue of the tensor $\left(\mathbf{S}^{2}+\boldsymbol{\Omega}^{2}\right)$, where $\mathbf{S}$ and $\boldsymbol{\Omega}$ are the symmetric and antisymmetric part of the velocity gradient tensor $\nabla \mathbf{U}$, respectively. The presence of a vortex core is associated with a negative value of $\lambda_{2}$. With reference to Fig. 4, which shows an isosurface of the $\lambda_{2}$ vortex identifier (the color indicates the sign of the axial vorticity of each vortex), we remind that the steady engulfment regime is characterized by two counter-rotating vortical structures which originate on the top wall of the mixer $(y=0.625)$, where an inclined (in a $x z$ plane) separation bubble forms at the confluence between the two incoming flows. Each vortical structure has a top part inside the separation bubble and two legs entering the outflow channel. For both structures, the two legs are asymmetric, their vorticity is almost aligned with the axis of the outflow channel ( $y$-direction) and one leg contains definitely more intense vorticity than the other. Moreover, the strong leg of one vortical structure is adjacent to the weak leg of the other vortical structure, so that at a very short distance after the beginning of the outflow channel, the two weak legs disappear and only the two strong legs persist. These two legs are formed by co-rotating vortices which break the reflectional symmetry of the flow and, consequently, strongly contribute to increase the mixing between the two streams entering the device.

The above described flow is characteristic of the steady engulfment regime but it is a typical vorticity configuration also in the UAR. Indeed, although the UAR is unsteady, it is characterized for the largest part of its period by a sequence of slowly evolving flow configurations that are close to the ER one. In order to describe the UAR dynamics, we report in Fig. 5 some flow snapshots properly distributed in an instability period showing the vortices, identified using the $\lambda_{2}$ criterion, together with the in-plane velocity field and the $y$-vorticity at some $y$-sections (Multimedia view). The distribution at selected $x z$ planes of a passive scalar entering from one of the two inlet channels is also reported in Fig. 5. Detailed views of the vortices at the confluence between the mixer channels are reported in Fig. 6. We will refer to those figures for the following description.

We arbitrarily choose Figs. 5(a) and 6(a) as the beginning of the instability cycle. The cycle thus starts with a flow configuration that is very similar to the engulfment regime, except for a rotation of the legs in the outflow pipe (point P1 in Fig. 5(a)) which will be explained later. As time progresses, the top parts of the vortical structures tend to approach each other. At the same time, the point P1 in 

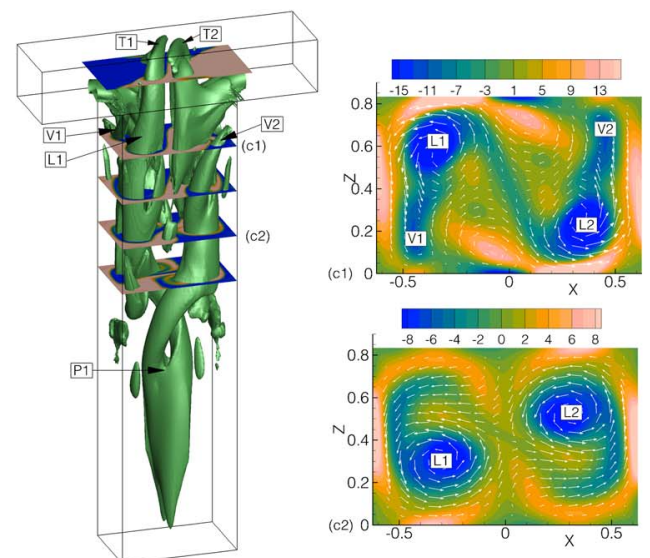

(a)
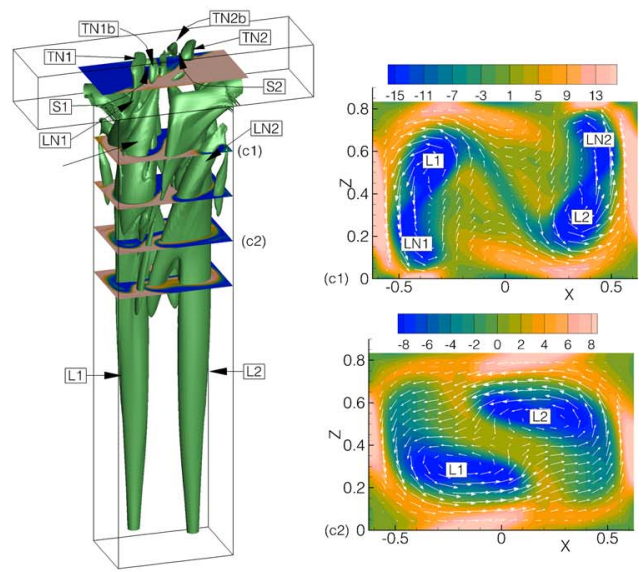

(b)
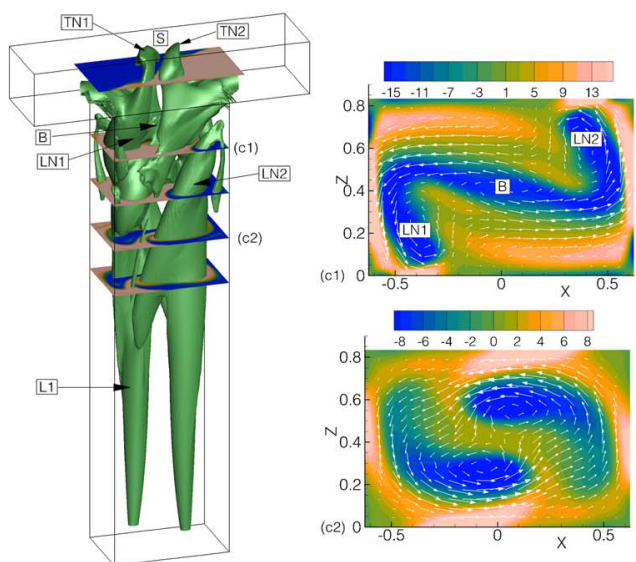

(c)
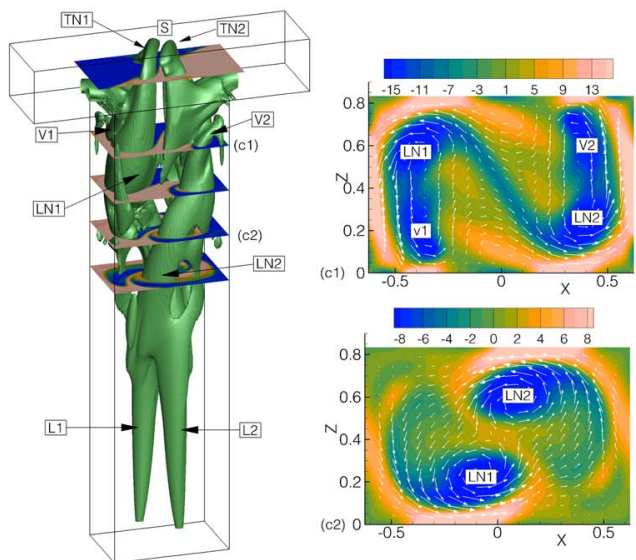

(d)

FIG. 5. UAR: flow snapshots at $R e=240$ taken at 4 significant phases of the instability cycle (from (a) to (d): $t / T \simeq 0,0.64$, $0.74,0.84, T$ being the flow period). Left figures show vortices identified by the $\lambda_{2}$ criterion and the distribution at some $y$-sections of a scalar passive entering from one of the two inlet channels; right figures show the in-plane velocity vectors and the normal $(y)$ vorticity (sections $y=-0.5$ and $y=-15$ ). (Multimedia view) [URL: http://dx.doi.org/10.1063/1.4885451.2]

Fig. 5(a) is convected in the outflow pipe so that the legs of the vortical structures become straight. This process is initially slow and the vortical structures on the top boundary (T1 and T2) of the mixer maintain their tilting angle. When the top parts of the two vortical structures become closer, their tilting angle rapidly increases (see Fig. 6(b)) up to a maximum value, at which they seem aligned with the mixing channel diagonal. At the same time, two new separation regions S1 and S2 form in the two inflow channels of the mixer (S1 and S2 in Fig. 5(b)) which are adjacent to the top corners of the mixer and in symmetric positions with respect to the $y$-axis. On each lateral face $(z=0$ and $z$ $=H$ ), the new separation regions form close to the strong legs L1 and L2 of the vortical structures. One of the two separation regions mentioned above is shown in Fig. 7, which is a detailed view of Fig. 5(b). As time passes, the two separation regions S1 and S2 travel towards the symmetry plane of the mixer, where they merge and replace the previous recirculation bubble associated with the top parts T1 and T2 of the initial vortical structure. As shown in Fig. 5(b), before merging, from S1 two new vortices of opposite sign starts to form, each one made by a top part (TN1 and TN1b) and a short leg. The same happens for S2 (TN2 and TN2b). The vortices which have the opposite sign with respect to the adjacent strong leg (TN1b and TN2b, respectively) quickly disappear and seem to play a minor role in the self-sustained process. Conversely, TN1 and TN2 start to grow in intensity and to generate two legs, LN1 and LN2, respectively, which elongate for convection in the 


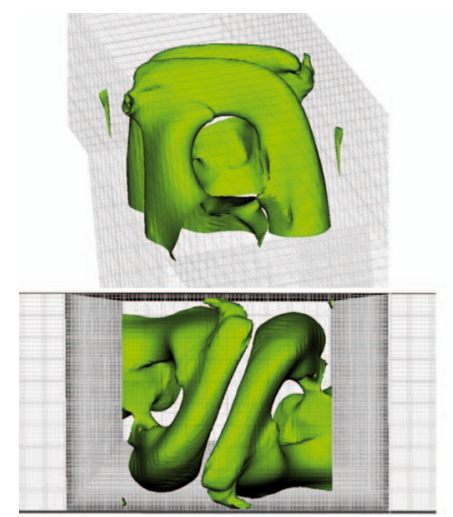

(a)

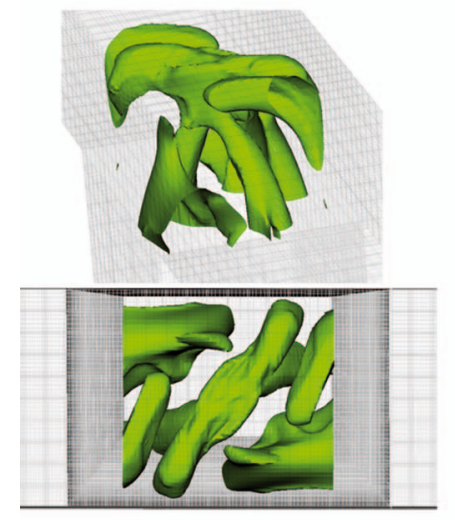

(c)

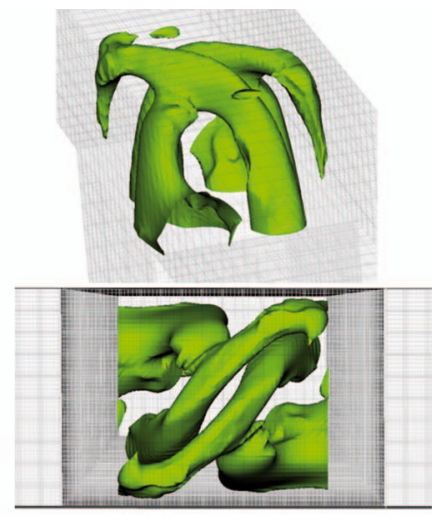

(b)

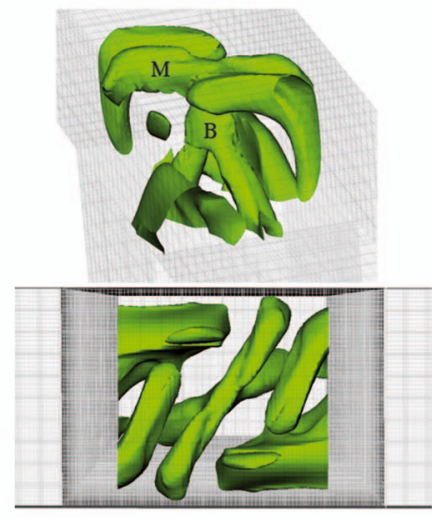

(d)

FIG. 6. Detailed view of vortices at the confluence between the three channels at properly selected phases (from (a) to (d): $t / T \simeq 0,0.45,0.61,0.65, T$ being the flow period).

$y$-direction as shown in Figs. 5(b) and 5(c). The leg LN1 (LN2) merges with a co-rotating vortex V1 (V2) of minor importance which is generated at the confluence between the inlet and the outflow channels, thus forming a continuous vortex leg (see Figs. 5(a) and 5(b)). Vortices V1 and V2 can also be observed in the engulfment regime for sufficiently high values of Re. At this point of the cycle, the top parts (T1 and T2) and the strong legs L1 and L2 of the two initial vortical structures merge, as shown in Fig. 6(c). The merged top parts (M in Fig. 6(d)), which remain located close to the top boundary of the mixer, contain vorticity of opposite sign and, hence, they quickly annihilate as well as the associated tilted recirculation bubble. Just after the merging (Fig. 6(d)), the two strong legs L1 and L2 are connected and, at their connection point a blob of vorticity (B) mainly aligned with the $y$-direction can be observed, which results from the sum of vorticity of equal sign. Starting from this moment, L1 and L2 are not fed anymore by the vorticity of the recirculation bubble on the top boundary of the mixer, since this bubble has disappeared. Thus, their intensity starts to quickly diminish. At the same time, the blob B is convected towards the outflow channel. A trace of the passage of B can be clearly seen in Fig. 5(c), at the center of section C1. As it is further convected in the outflow channel its intensity decreases rapidly, as that of L1 and L2. At the same time, the legs LN1 and LN2 travels towards the center of the channel together with their source vorticity regions $\mathrm{S} 1$ and $\mathrm{S} 2$, until S1 and S2 merge in a single separation region S and TN1 and TN2 replace T1 and T2, as shown in Figs. 5(c) and 5(d). At this stage, the strong legs of the vortical structures are made by two contributions, LN1 and L1 for the first vortex, LN2 and L2 for the second one, as illustrated in Figs 5(c) and 5(d). This fact explains the peculiar shape of the legs of the vortices in the considered phase of the instability cycle (see Fig. 5(d)). The described process is repeated periodically. 

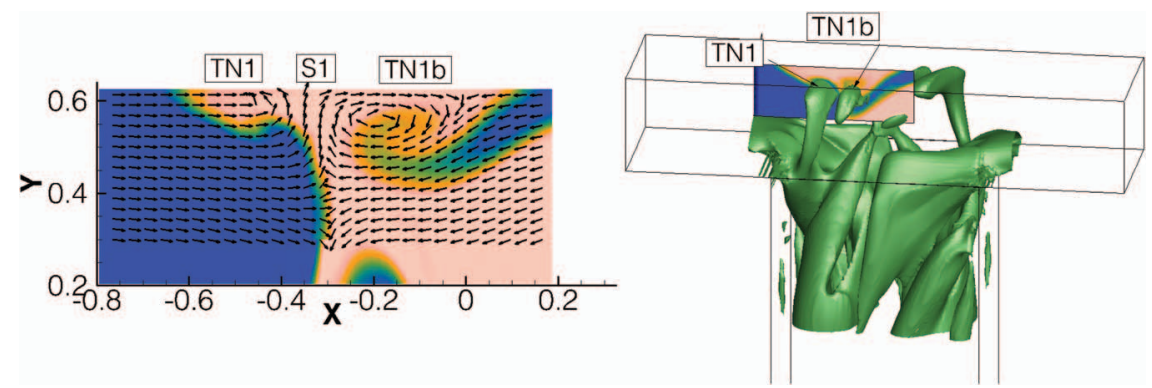

FIG. 7. Scalar passive and in-plane velocity field (left figure) at the z-section reported on the right figure at the same phase as in Fig. 5(b).

Looking at the normal vorticity dynamics in $x z$-sections in the mixing channel, the typical configurations observed in a UAR cycle are the following: a configuration similar to that of the steady engulfment regime, in which the two co-rotating vortices (L1 and L2) are well separated (see sections in Fig. 5(a)). Successively, the intensity of the two vortices start to decrease and a new couple appears (LN1 and LN2) with an increasing intensity (see sections in Fig. 5(b)). Finally, L1 and L2 disappear and they are replaced by LN1 and LN2. If the considered section is close to the beginning of the outflow channel, as for section $\mathrm{C} 1$ in Fig. 5, L1 and L2 disappear by merging at the center of the channel, and this is the blob B, described above, which crosses the considered section (see Fig. 5(c), section C1). At a larger distance from the beginning of the outflow channel, as for section C2, L1 and L2 disappear more smoothly without observing the blob B (see Figs. 5(c) and 5(d)) and they are replaced by LN1 and LN2, respectively.

As previously pointed out, at the confluence between the two inlet channels, vortices resembling a Kelvin-Helmholtz instability form, approach and disappear, this process being repeated periodically in time. These have been visualized in Fig. 3 where the dynamics of a passive scalar entering from one of the two inlet channels is shown at section $y=0.31$. The same section is reported in Fig. 5 in order to show that the above mentioned vortices are not related to a Kelvin-Helmholtz instability but they are the trace of the typical vortical structures of the UAR regime at plane $y=0.31$. This can be seen even more clearly by comparing Figs. 8(a) and 8(b), which are zoomed and elaborated views of Fig. 5(a).

\section{B. Unsteady symmetric regime}

As already pointed out, the flow regime observed in the range $440 \leq R e \leq 480$ is the USR. In the USR, the flow passes continuously and periodically between a symmetric state, similar to the steady vortex regime, and an asymmetric state, similar to the steady engulfment regime but

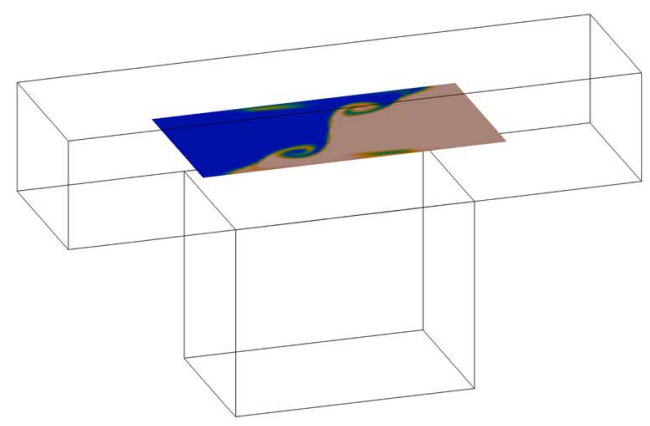

(a)

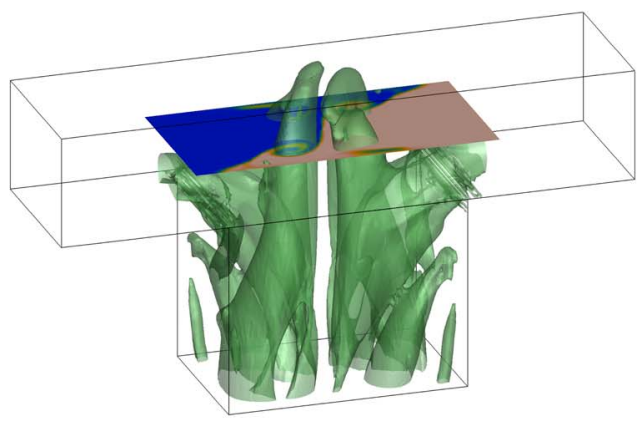

(b)

FIG. 8. Detailed view of vortices at the confluence between the two inlet channels at phase $t / T=0$ : scalar passive distribution at section $y=0.31$ (a) without and (b) with the visualization of the vortices. 


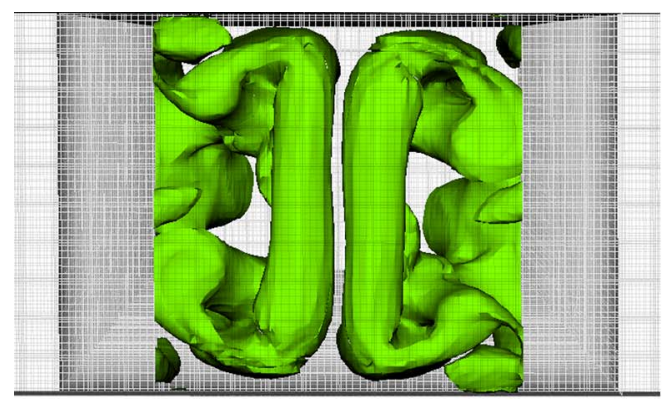

(a)

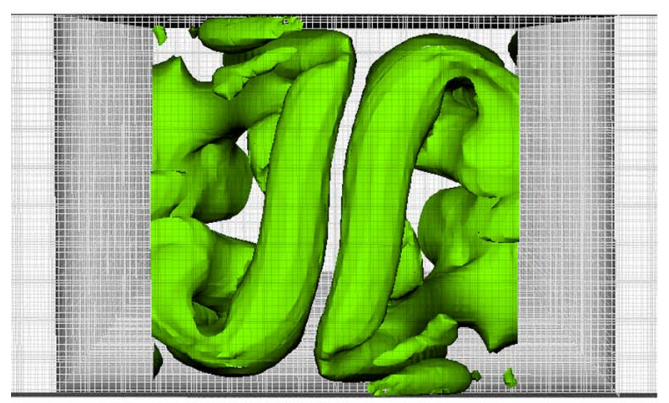

(c)

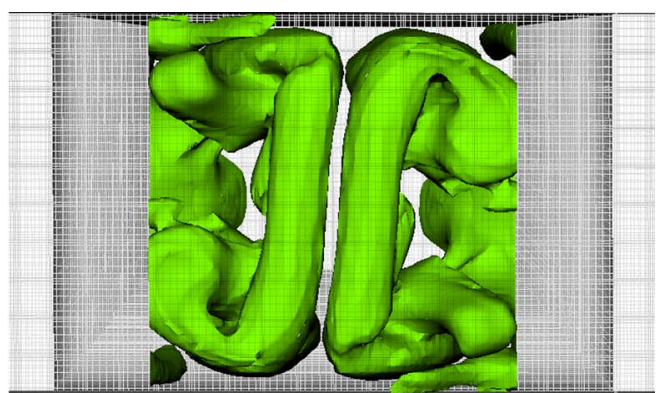

(b)

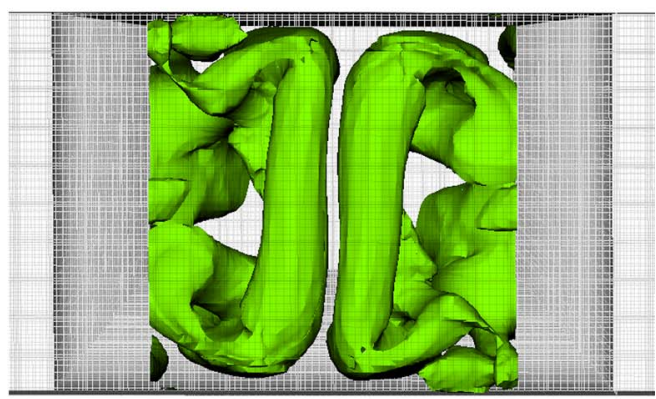

(d)

FIG. 9. (a)-(d) USR ( $R e=480)$ : top view of the vortices ( $\lambda_{2}$ criterion) at the confluence between the three channels of the mixer at 4 phases uniformly distributed in one cycle of the flow regime.

with a smaller tilting angle of the separation bubble on the top boundary of the mixer, and, hence, of the top parts of the 3D vortical structures. This is shown in Fig. 9, where a top view of the vortical structures identified by the $\lambda_{2}$ criterion is reported for (a) the instant in which the separation surface between the two incoming flows in the inlet channels is symmetrical and the instant (c) of maximum tilting angle. The flow configuration in Fig. 9(a) resembles the steady vortex regime, as it is characterized by two almost symmetric counter-rotating vortical structures each one made by two almost symmetric legs of equal intensity. The flow configuration in Fig. 9(c) resembles the ER with a small tilting angle, and each vortical structure is now made by two asymmetric legs, a strong one and a weak one. The flow switches smoothly from Figs. 9(a) to 9(c) and returns to Fig. 9(a) in one period.

The vorticity in the legs of the vortical structures, which originates from the confluence region between the streams entering the device, is convected in the outflow channel by the flow field. Thus, as a consequence of the previously described dynamics of the top parts of the vortical structures, in each $x z$-section of the outlet channel the flow periodically and progressively switches from a symmetric configuration (with respect to the plane $x=0$ ), made by two couples of counter-rotating vortices, typical of the steady vortical regime, to an asymmetric configuration, made by two corotating vortices in specular position with respect to the axis of the outlet channel, typical of ER. This is illustrated in Fig. 10, where the 3D vorticity structures are plotted together with two $x z-$ sections of the in-plane velocity field and of the $y$-vorticity component (Multimedia view). The description of the USR given here is in full agreement with that given by Thomas and Ameel ${ }^{13}$ and Thomas et al. ${ }^{14}$

\section{Stability and sensitivity analyses}

In this section, we focus on the onset and on the characteristics of the asymmetric periodic regime by using stability and sensitivity analysis. It has been shown previously that, as $R e$ is increased, this is the first unsteady flow regime observed in the mixer. 


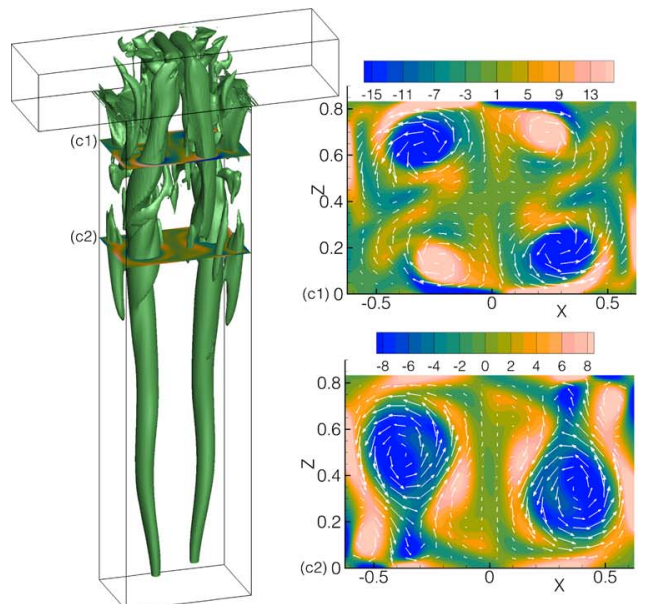

(a)

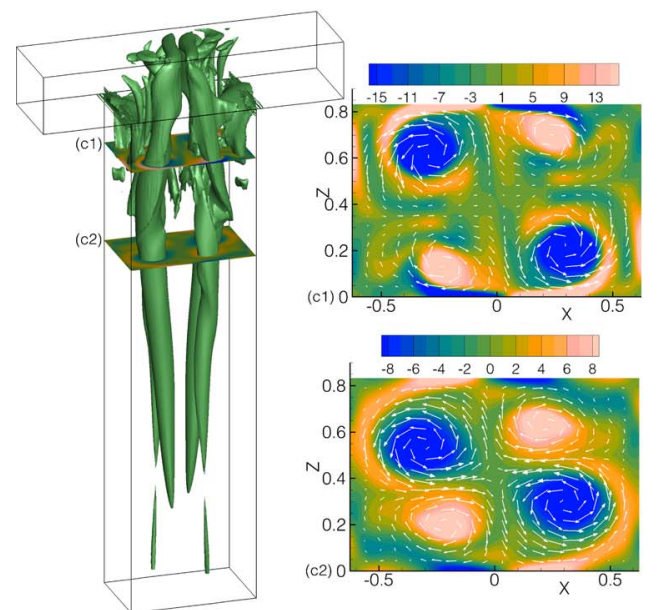

(c)

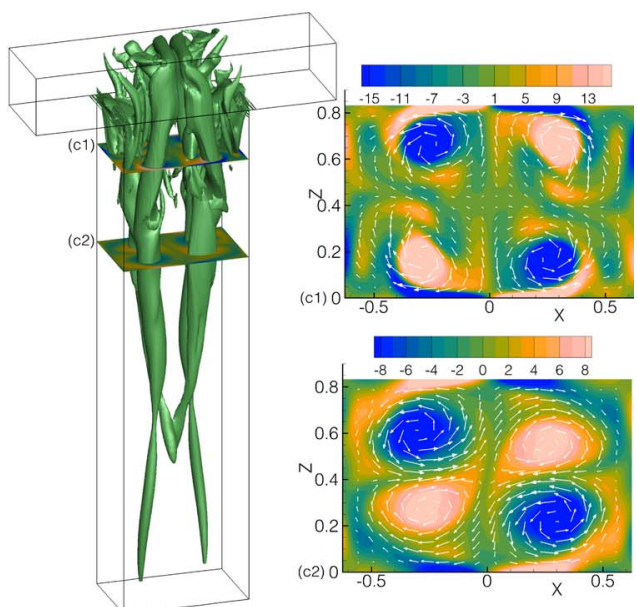

(b)

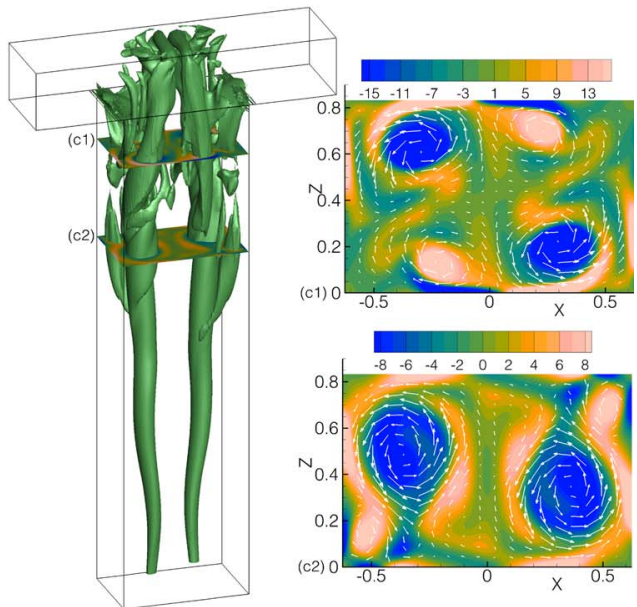

(d)

FIG. 10. (a)-(d) USR ( $R e=480)$ : vortices $\left(\lambda_{2}\right.$ criterion) at 4 phases uniformly distributed in one cycle of the flow regime, together with in-plane velocity and $y$-vorticity at two selected $x z$-sections $(y=-0.5$ and $y=-1.5)$. (Multimedia view) [URL: http://dx.doi.org/10.1063/1.4885451.3]

The steady base flow, which might be unstable for Reynolds numbers larger than the critical one ( $R e \simeq 220$ from DNS), is computed for the stability analysis by using the selective frequency damping method in a time-marching procedure. ${ }^{19}$

For a given value of $R e$, the global stability of the base flow is investigated by looking for the leading global mode $(\hat{\mathbf{u}}, \hat{p})$, defined as the global mode of largest growth rate. The computed eigenvalue $\sigma$ for three different Reynolds numbers is given in Table I, showing that the instability onset occurs in the range $220<R e_{c}<230$, in agreement with the DNS results described in Sec. III. The leading eigenmode is complex valued and it is linked to an Hopf bifurcation, as it could be expected on the basis of the DNS flow analysis. The Strouhal numbers predicted by the stability analysis are in agreement with the ones evaluated from DNS, also reported in Table I for reference. In particular, the Strouhal numbers are in excellent agreement at $R e=230$, with discrepancies of the order of $1 \%$, while at $R e=240$ discrepancies grow to $9 \%$, as reasonable when Reynolds numbers progressively larger than the critical one are considered, because of the increasing importance of nonlinearities.

Fig. 11 shows a three-dimensional view of the leading eigenmode, depicted by an isosurface of the magnitude of the real part of the velocity field, computed at $R e=220$. It can be seen that 
TABLE I. Leading eigenvalue $\sigma$ and associated Strouhal number $S t$ for various Reynolds numbers.

\begin{tabular}{lrcc}
\hline \hline Re & $\sigma$ & $S t$ & $S t(\mathrm{DNS})$ \\
\hline 220 & $-4.47 \times 10^{-2}+0.971 i$ & 0.155 & $\ldots$ \\
230 & $2.33 \times 10^{-2}+0.997 i$ & 0.159 & 0.16 \\
240 & $9.29 \times 10^{-2}+1.028 i$ & 0.164 & 0.18 \\
\hline \hline
\end{tabular}

the global mode is localized at the confluence between the two streams and in the mixing channel. According to the modal decomposition used for the stability analysis, the three-dimensional unsteady flow $\mathbf{U}$ (in conditions of marginal stability) can be reconstructed using the base flow $\mathbf{U}_{b}$ and the global mode $\hat{\mathbf{u}}$

$$
\mathbf{U}(t)=\mathbf{U}_{\mathbf{b}}+A(\Re(\hat{\mathbf{u}}) \cos (\omega t)-\mathfrak{J}(\hat{\mathbf{u}}) \sin (\omega t))
$$

where $\mathfrak{R}(a)$ and $\mathfrak{J}(a)$ are, respectively, the real and imaginary part of the complex field $a$. The amplitude of the perturbation $A$ cannot be obtained by the linear analysis, but it can be estimated from post-processing of the available DNS data. Fig. 12 shows three different snapshots of the reconstructed unsteady flow at $R e=230$ close to the confluence of the channels. The vortical structures, depicted using the $\lambda_{2}$ criterion, are in excellent agreement with the ones from the DNS described above in Sec. III (see Fig. 6).

The adjoint mode is shown in Fig. 13, by the same velocity isosurface as in Fig. 11. It is evident that the adjoint mode is located only in the inlet channels. Therefore, the instability core, given by the product $\left\|\mathbf{u}^{+}\right\|\|\hat{\mathbf{u}}\|$ is localized entirely in the T confluence, as shown in Fig. 14(a). As highlighted in Giannetti and Luchini, ${ }^{16}$ this scalar field is non-negligible in the region where the feedback process responsible of a self-sustained periodic instability is active. In particular, the frequency and the onset of the instability strongly depend on the details of the flow inside the instability core. Fig. 14(a) shows that the core of the unsteady asymmetric instability is localized in the region where the two inlet streams meet and the co-rotating vortices are periodically formed (see, e.g., Fig. 14(b) in which an $x z$ section of the instability core is superposed to the trace of the vortices on the considered plane). Note also that the instability core is asymmetric, because the steady flow before the onset of the instability is the one characteristic of the steady engulfment regime, which is also asymmetric.

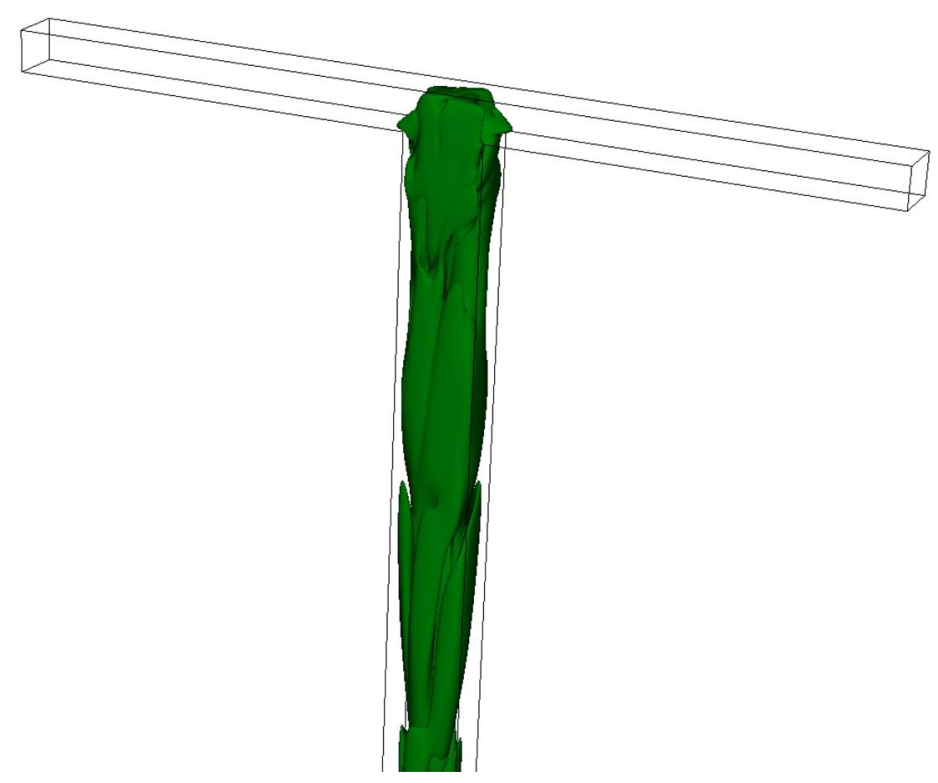

FIG. 11. Global eigenmode, depicted by an isosurface at a small threshold value of the magnitude of the real part of the velocity field, computed at $R e=220$. 


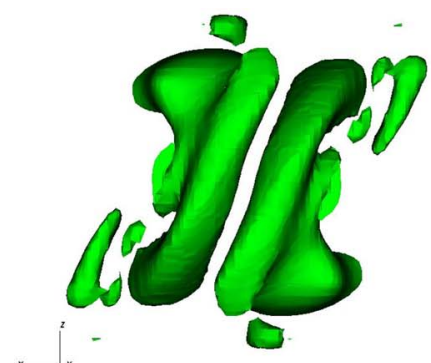

(a)

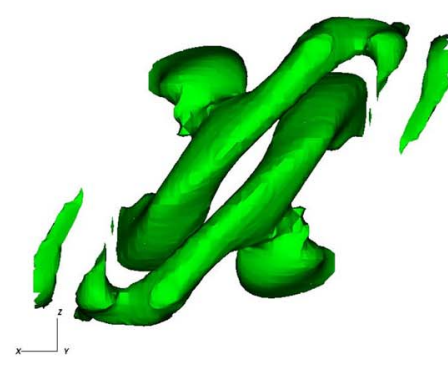

(b)

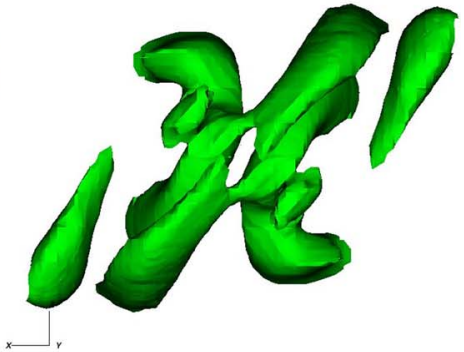

(c)

FIG. 12. Stability analysis: reconstruction of the three-dimensional flow $\mathbf{U}(t)$ at three different instant (a)-(c) along the instability cycle. Vortical structures are identified through the $\lambda_{2}$ criterion.

Since the asymmetry of the engulfment regime is due to a pitchfork bifurcation, the asymmetry can appear in two possible positions, characterized by opposite tilting angles. Depending on small imperfections of the geometry or of the flow conditions, one or the other configuration may occur.

The sensitivity of the considered instability with respect to a generic perturbation of the baseflow can be computed following Eq. (3). This sensitivity, which is represented by the vector field $M^{+}$in Eq. (4), is shown in Fig. 15(a), identified by an isosurface of its magnitude. Note that, as for the instability core, the sensitivity is localized in the overlapping region between the direct and the adjoint modes, thus where the three channels meet. Therefore, this zone is the one in which a modification of the base flow would be most effective in modifying, promoting, or delaying the instability leading to the unsteady regime. Modifications of the base flow can be obtained by active or passive ${ }^{21}$ flow controls. Moreover, $M^{+}$is a complex field and its real and imaginary parts give the sensitivity to base-flow modifications of the growth rate and of the frequency, respectively. As an example, these sensitivity fields are plotted separately in Figs. 15(b) and 15(c) at the $y$-section $y=0.4$. From these figures, it is evident that the flow is sensitive to base-flow modifications close to the tilted vortices at the confluence between the inlet channels. In particular, Fig. 15(b) shows that a base-flow modification which induces a reduction of the tilting angle, has a stabilizing effect, i.e., it produces a negative variation of the eigenvalue ( $\delta \sigma$ in Eq. (3)). Clearly, the opposite effect is obtained for a modification which induces an increase of the vortical structures tilting angles. Fig. 15(c) indicates that the largest variation of the instability frequency is obtained for a base-flow modification by acting on the parts of the 3D vortical structures which become the strong co-rotating legs in the mixer-channel; the frequency grows if the tilt angle is increased.

As previously said, the information provided by the sensitivity maps can be used to design control strategies, aimed at promoting or delaying the flow instability. As an example, we focus, herein, on the effect of a modification of the incoming velocity profile. The sensitivity of the instability to a modification of the incoming velocity profile can be computed through Eq. (7) and thus, it involves both the adjoint base flow pressure $P_{b}^{+}$and a viscous contribution weighted by the inverse of the Reynolds number. The latter term has been found to be negligible, which means

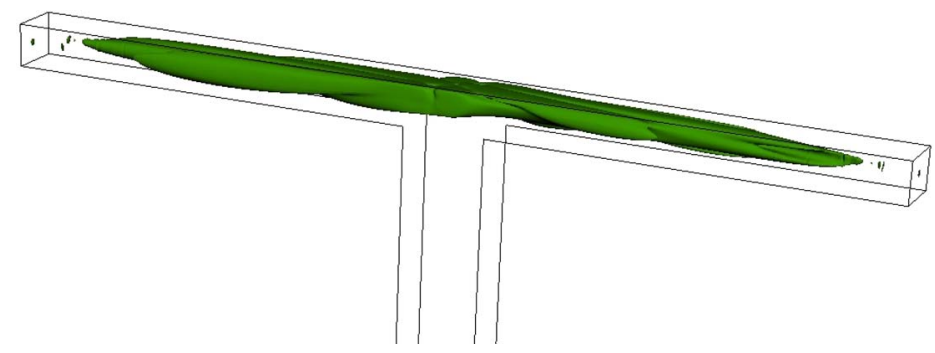

FIG. 13. Adjoint mode, depicted by an isosurface at a small threshold value of the magnitude of the real part of the velocity field, computed at $R e=220$. 


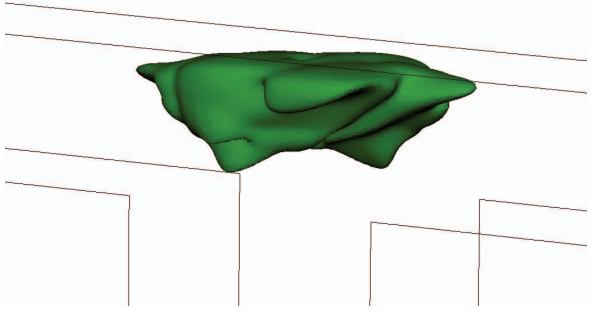

(a)

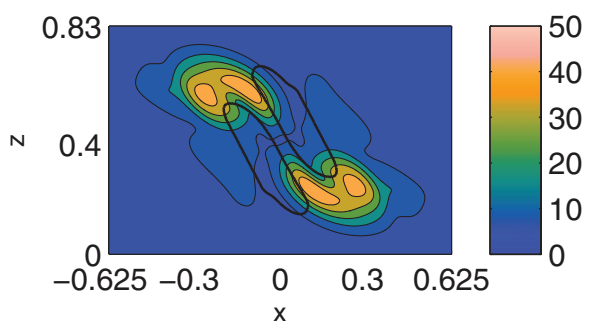

(b)

FIG. 14. Instability core $\left\|\mathbf{u}^{+}\right\|\|\hat{\mathbf{u}}\|$ at $R e=220$. (a) Three-dimensional view and (b) slice at $y=0.5$. The base-flow vortices in (b) are identified (tick line) by using the $\lambda_{2}$ criterion.

that the flow is sensitive almost only to a perturbation of the component of the velocity normal to the inflow boundary, since $P_{b}^{+}$acts only in this direction. Therefore, if we consider a localized perturbation of the form $\delta \mathbf{U}_{\mathbf{i}}=U_{i} \delta(x, z) \mathbf{n}, \delta(x, z)$ being the two-dimensional Dirac delta function and $\mathbf{n}$ the normal unit vector to the inlet boundary pointing outside the flow domain, Eq. (7) can be written as $\delta \sigma=U_{i} S(x, z), S$ being a two-dimensional sensitivity function of the eigenvalue to a localized modification of the normal component of the inflow velocity. A similar analysis was carried out in Fani $e t a l .{ }^{8}$ for the instability leading to the engulfment regime, showing that with a flat inlet velocity profile the engulfment onset occurs at larger Reynolds numbers than for a fully developed profile. The sensitivity function $S$ obtained in the present case is depicted in Fig. 16 for the two inlet sections $x=-L_{i}$ and $x=L_{i}$. The maps at the first inlet, plotted in Figs. 16(a) and 16(c), are related to second inlet ones, depicted in Figs. 16(b) and 16(d), through a reflection around the plane $z=H / 2$. In particular, Figs. 16(a) and 16(b) show the real part of $S$, i.e., that related to the eigenvalue growth factor. As a first comment, the sign of the growth factor sensitivity in Figs. 16(a) and 16(b) is always negative. This implies that a localized decrease of the inflow velocity, whatever area is

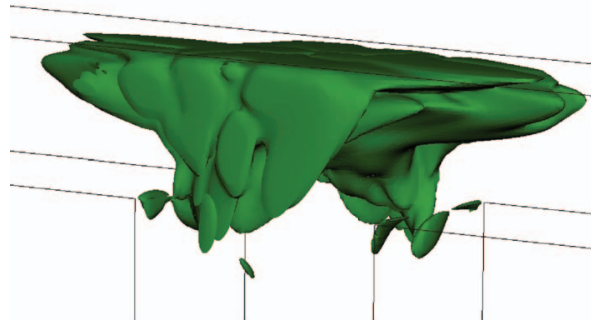

(a)

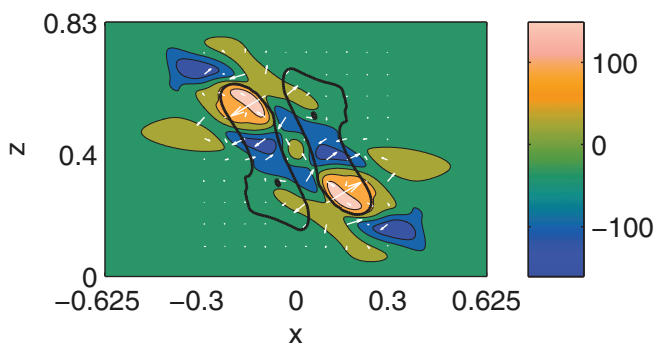

(b)

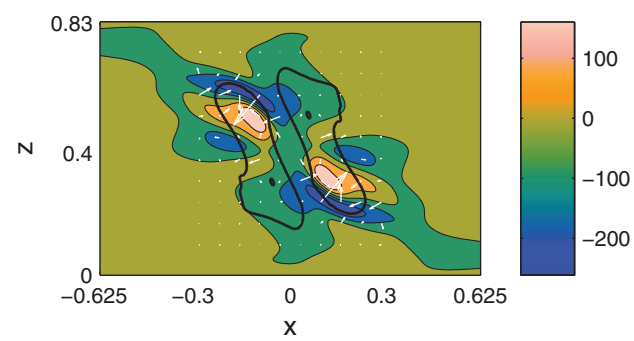

(c)

FIG. 15. (a) Three-dimensional view of the sensitivity to base flow modifications magnitude. Slice at $y=0.4$ of the growth rate (b) and frequency sensitivity (c) to base-flow modification: arrows indicate the in-plane sensitivity components, whose maximum magnitude is approximately equal to 800 (b) and 1000 (c), while contours represent the sensitivity component normal to the plane.The base-flow vortices are identified (tick line) by using the $\lambda_{2}$ criterion. 


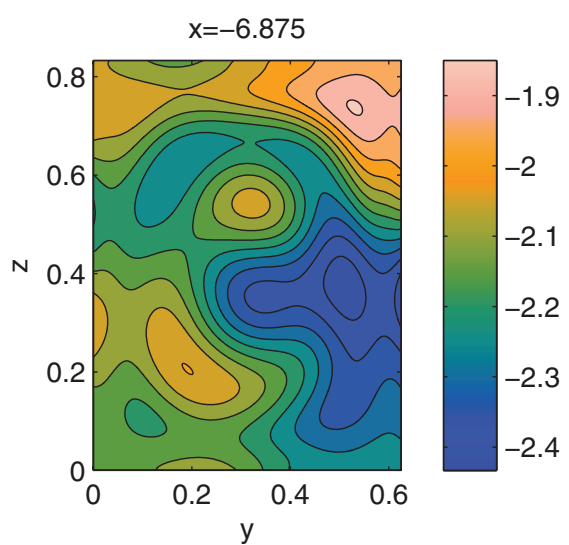

(a)

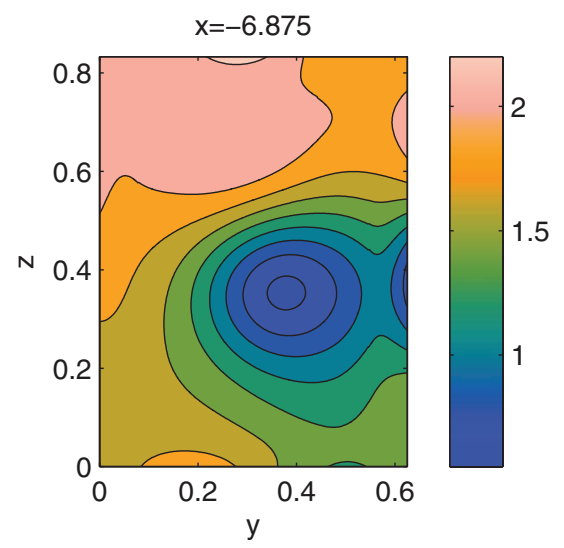

(c)

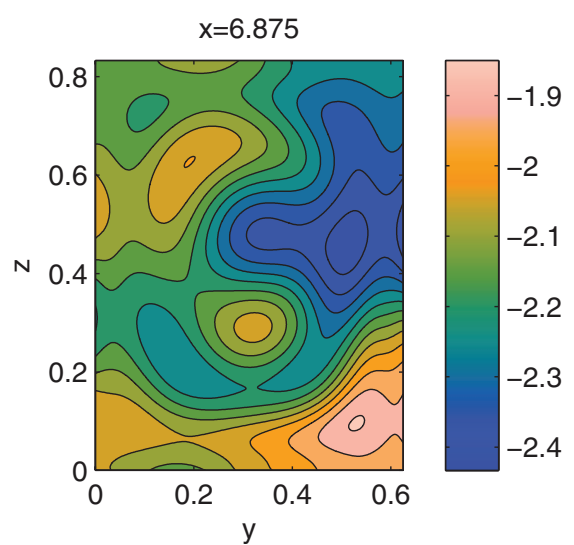

(b)

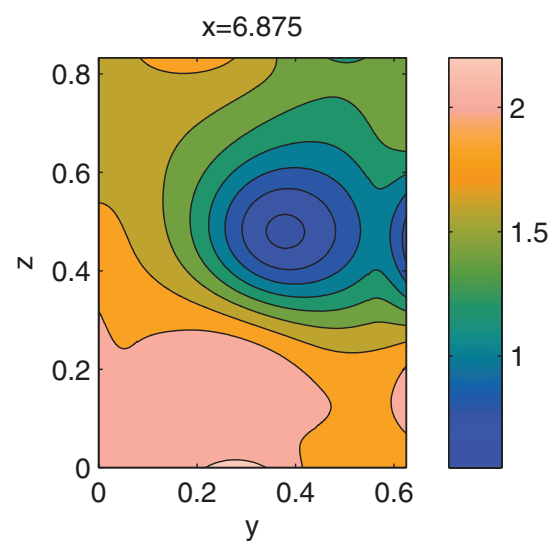

(d)

FIG. 16. Sensitivity to modifications of the velocity profile normal to the inlet plane at $R e=220$, computed at the two inlet surfaces. Growth rate (a) and (b) and frequency (c) and (d) sensitivities.

considered, always implies a negative shift of the eigenvalue, i.e., a stabilizing effect. However, the sensitivity is not uniform but it is higher close to the top wall of the mixer $(y=W)$ and in the lower $(z<H / 2)$ or upper $(z>H / 2)$ part of the section, depending on which inlet boundary we consider. Consequently, it is possible to perturb the inflow profile so as to preserve the mass flow rate and, thus, the Reynolds number but, at the same time, to have an effect on the instability growth rate. For instance, as for the ER regime, with a flat (e.g., a non-fully developed) inlet velocity profile the UAR onset occurs at larger Reynolds numbers than for a fully developed profile. As concerns the mode frequency, its modification is related to the imaginary part of $S$ shown in Figs. 16(c) and 16(d) for the two inlet sections. A localized decrease (increase) of the inflow velocity at a generic location of both inflow boundaries always leads to a decrease (increase) of the instability frequency. This is consistent with the fact that in the UAR regime the Strouhal number increases as $R e$ is increased (see Fig. 2). The effect of a perturbation of the inlet velocity on the instability frequency is more intense if the perturbation is given on the parts of the 3D vortical structures which become the strong co-rotating legs in the mixer-channel (for large values of $z$ in the section at $x=-L_{i}$ and for small $z$ at $x=L_{i}$ ). This is consistent with the previous analysis of Fig. 15(c).

\section{CONCLUSIONS}

The unsteady flow regimes occurring in a T-mixer configuration have been investigated by means of direct numerical simulations. In agreement with experimental findings for a similar Tmixer configuration, we identified two distinct time-periodic regimes. 
The first one, occurring for $230<R e<400$, is asymmetric in space and periodic in time with a frequency monotonically increasing with the Reynolds number. This regime, denoted as UAR has been characterized in terms of the dynamics of 3D vortical structures forming at the confluence between the two streams entering the T-mixer. In particular, we showed that co-rotating vortices resembling a Kelvin-Helmholtz instability, observed on $x z$ planes at the interface between the two streams, are actually the traces of 3D vortical structures. These structures, which are similar to those also present in the steady engulfment regime, have a top part inside the separation bubble forming at the stream confluence, which are tilted with respect to the inlet flow direction, and two asymmetric legs entering the outflow channel. For each structure, only the leg containing the more intense vorticity survives moving downstream in the mixing channel, giving rise to two co-rotating vortices responsible for the engulfment regime and for the related mixing enhancement. In the UAR, these structures undergo a cyclic behavior: the two top parts of the 3D structures approach each other, as the top parts become closer, their tilting angle rapidly increases up to a maximum value, at which the top parts and the strong legs of the two vortical structures merge. The top part, containing vorticity of opposite sign, annihilate after merging, while the strong legs create a blob of vorticity, which is quickly convected towards the outflow channel; starting from this moment the legs are not fed anymore and their intensity quickly decreases. In the same time, two new 3D structures of the same type form and are convected towards the symmetry plane, where they will replace the original two vortical structures. The cycle then starts again.

By further increasing the Reynolds number $(440 \leq R e \leq 480)$, again in agreement with the experimental findings, a different time-periodic regime is observed (USR), characterized by a continuous and periodic switch from a symmetric configuration to a slightly asymmetric one that resembles the engulfment regime.

Besides DNS, three-dimensional linear stability analysis has been used, for the first time to our knowledge, to characterize the instability leading to the UAR, which is also interesting for applications due to the high mixing efficiency of this regime. The unstable mode was found to be complex valued and it is linked to an Hopf bifurcation, as it could be expected on the basis of the DNS flow analysis. The critical Reynolds number and the instability frequency computed through the instability analysis are in very good agreement with those found in DNS, as well as for the vorticity distribution reconstructed by summing the base flow and the unstable global mode.

The instability was further characterized by a sensitivity analysis, which allows the identification of the instability core; this has been found to be located close to the confluence region between the inlet channels. Indeed, consistently with the flow dynamics observed in DNS, the core of the instability coincides with the region where the vortical structures typical of the analyzed flow regime form. This information is useful to design control strategies aimed at promoting or delaying the onset of the UAR. In particular, the sensitivity analysis has been specialized here to investigate the effect of a perturbation of the inlet velocity profile. Similar to what previously observed for the onset of the steady engulfment regime, it can be concluded that for a flat (e.g., non-fully developed) inlet velocity profile the UAR onset is delayed at larger Reynolds numbers than for a fully developed profile.

\section{ACKNOWLEDGMENTS}

The authors wish to thank CINECA Computing Center (Bologna, Italy) for allowance of computational resources on FERMI supercomputer under the ISCRA program.

${ }^{1}$ D. Bothe, C. Stemich, and H. J. Warnecke, "Fluid mixing in a T-shaped micro-mixer," Chem. Eng. Sci. 61, 2950-2958 (2006).

${ }^{2}$ M. Hoffmann, M. Schlüter, and N. Räbiger, "Experimental investigation of liquid-liquid mixing in T-shaped micro-mixers using $\mu$-LIF and $\mu$-PIV," Chem. Eng. Sci. 61, 2968-2976 (2006).

${ }^{3}$ A. Soleymani, H. Yousefi, and I. Turunen, "Dimensionless number for identification of flow patterns inside a T-micromixer," Chem. Eng. Sci. 63, 5291-5297 (2008).

${ }^{4}$ S. K. R. Cherlo and S. Pushpavanam, "Effect of depth on onset of engulfment in rectangular micro-channels," Chem. Eng. Sci. 65, 6486-6490 (2010).

${ }^{5}$ A. Soleymani, H. Yousefi, W. Ratchananusorn, and I. Turunen, "Pressure drop in micro T-mixers," J. Micromech. Microeng. 20, 015029 (2010). 
${ }^{6}$ C. Galletti, M. Roudgar, E. Brunazzi, and R. Mauri, "Effect of inlet conditions on the engulfment pattern in a T-shaped micro-mixer," Chem. Eng. J. 185-186, 300-313 (2012).

${ }^{7}$ G. Orsi, M. Roudgar, E. Brunazzi, C. Galletti, and R. Mauri, "Water-ethanol mixing in T-shaped microdevices," Chem. Eng. Sci. 95, 174-183 (2013).

${ }^{8}$ A. Fani, S. Camarri, and M. V. Salvetti, "Investigation of the steady engulfment regime in a three-dimensional T-mixer," Phys. Fluids 25, 064102 (2013).

${ }^{9}$ R. J. Poole, M. Alfateh, and A. P. Gauntlett, "Bifurcation in a T-channel junction: Effects of aspect ratio and shear-thinning," Chem. Eng. Sci. 104, 839-848 (2013).

${ }^{10}$ R. J. Santos and M. A. Sultan, "State of the art of mini/micro jet reactors," Chem. Eng. Technol. 36, 937-949 (2013).

${ }^{11}$ S. Dreher, N. Kockmann, and P. Woias, "Characterization of laminar transient flow regimes and mixing in T-shaped micromixers," Heat Transfer Eng. 30, 91-100 (2009).

${ }^{12}$ A. Minakov, V. Rudyak, A. Dekterev, and A. Gavrilov, "Investigation of slip boundary conditions in the t-shaped microchannel," Int. J. Heat Fluid Flow 43, 161-169 (2013).

${ }^{13} \mathrm{~S}$. Thomas and T. A. Ameel, "An experimental investigation of moderate Reynolds number flow in a T-channel," Exp. Fluids 49, 1231-1245 (2010).

${ }^{14}$ S. Thomas, T. A. Ameel, and J. Guilkey, “Mixing kinematics of moderate Reynolds number flows in a T-channel," Phys. Fluids 22, 013601 (2010).

${ }^{15}$ A. Bottaro, P. Corbett, and P. Luchini, "The effect of base flow variation on flow stability," J. Fluid Mech. 476, 293-302 (2003).

${ }^{16}$ F. Giannetti and P. Luchini, "Structural sensitivity of the first instability of the cylinder wake," J. Fluid Mech. 581, 167-197 (2007).

${ }^{17}$ O. Marquet, D. Sipp, and L. Jacquin, "Sensitivity analysis and passive control of cylinder flow," J. Fluid Mech. 615, 221-252 (2008).

${ }^{18}$ P. F. Fisher, "An overlapping Schwarz method for spectral element solution of the incompressible Navier-Stokes equations," J. Comput. Phys. 133, 84-101 (1997).

${ }^{19}$ E. Åkervik, L. Brandt, D. S. Henningson, J. Hœpffner, O. Marxen, and P. Schlatter, "Steady solutions of the Navier-Stokes equations by selective frequency damping," Phys. Fluids 18, 068102 (2006).

${ }^{20}$ J. Jeong and F. Hussain, "On the identification of a vortex," J. Fluid Mech. 285, 69-94 (1995).

${ }^{21}$ A. Fani, S. Camarri, and M. V. Salvetti, "Stability analysis and control of the flow in a symmetric channel with a sudden expansion,” Phys. Fluids 24, 084102 (2012). 